\title{
FSCN1 Promotes Epithelial-Mesenchymal Transition Through Increasing Snail1 in Ovarian Cancer Cells
}

\author{
Jie $\mathrm{Li}^{\mathrm{a}}$ Songlin Zhang ${ }^{\mathrm{b}}$ Meili Peia Lei Wu ${ }^{\mathrm{a}}$ Yanli Liu ${ }^{\mathrm{a}}$ Huijin Lic Jiaojiao Lu ${ }^{\mathrm{d}}$ \\ $\mathrm{Xu} \mathrm{Lid}$
}

aDepartment of Gynecology and Obstetrics, the First Affiliated Hospital of Xi'an Jiaotong University, Xi'an, 'Department of Structural Heart Disease, the First Affiliated Hospital of Xi'an Jiaotong University, Xi'an, 'Shaanxi Key Laboratory of Ischemic Cardiovascular Disease, Institute of Basic and Translational Medicine, Xi'an Medical University, Xi'an, ${ }^{\mathrm{C} C e n t e r}$ for Translational Medicine, the First Affiliated Hospital of Xi'an Jiaotong University, Xi'an, China

\section{Key Words}

FSCN1 • Snail1 • Ovarian cancer • Epithelial-mesenchymal transition

\begin{abstract}
Background/Aims: Epithelial-mesenchymal transition (EMT) is one of the key mechanisms mediating cancer progression. Snail1 has a pivotal role in the regulation of EMT, involving the loss of E-cadherin and concomitant upregulation of vimentin, among other biomarkers. We have found FSCN1 promoted EMT in ovarian cancer cells, but the precise mechanism of FSCN1 in EMT process has not been clearly elucidated. Methods: The levels of FSCN1 and snail1 were determined in epithelial ovarian cancer(EOC) specimen and in ovarian cancer cells by RT-qPCR. The changes of EMT makers and effects on snail1 by FSCN1 were examined by overexpression or depletion of FSCN1 in EOC cells by RT-qPCR and western blotting. The invasiveness of the FSCN1-modified EOC cells was examined in transwell assay. Co-immunoprecipitation (IP) was performed to detect the interaction between snail 1 and FSCN1 in EOC cells. Results: We found FSCN1 and snail 1 significantly increased in EOC, and especially in EOC with metastasis. FSCN1 was positively correlated with snail1 expression at the cellular/histological levels. Moreover, we further showed that FSCN1 physiologically interacted with and increased the levels of snail 1 to promote ovarian cancer cell EMT. Conclusion: FSCN1 promote EMT through snail1 in ovarian cancer cells. FSCN1 is an attractive novel target for inhibiting invasion and metastasis of EOC cells.
\end{abstract}




\section{Cellular Physiology Cell Physiol Biochem 2018;49:1766-1777

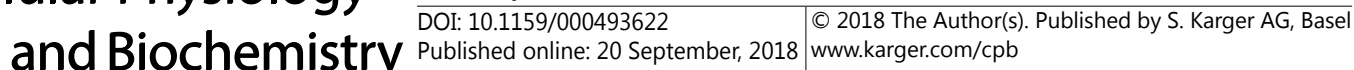 \\ Li et al.: Interaction of FSCN1 and Snail1 in EOC}

\section{Introduction}

Ovarian cancer is the most lethal gynecological tumor and ranks the fifth in the cause of death for women suffered from tumor [1,2]. The poor prognosis of ovarian cancer patients is mainly attributed to cancer metastasis, which highlights the importance of the elucidation of the molecular control of migration and invasiveness of ovarain cancer cells.

Epithelial-mesenchymal transition (EMT) is a process by which epithelial cells acquire a migratory, mesenchymal phenotype, characterized by loss of epithelial markers, acquisition of mesenchymal molecules and enhancement of cell mobility [3-5]. Loss of E-cadherin is considered to be a fundamental event in EMT process. Transcription factors that repress E-cadherin are considered as EMT-promoting factors, including snail1, slug, ZEB1, ZEB2, E47 and Kruppel-like factor 8 (KLF8) that directly bind to E-cadherin promoter and repress its transcription, and including twist, TCF4, SIX1 and FOXC2 that repress E-cadherin indirectly [4-6]. Snail1 has a pivotal role in the regulation of EMT, involving the loss of E-cadherin and claudins with concomitant upregulation of vimentin and fibronectin, among other biomarkers [7-9].

FSCN1 is an actin-bundling protein that plays a crucial role in cell movement under physiological or pathological conditions [10]. FSCN1 has been reported that its expression is associated with clinically unfavorable phenotypes in various human epithelial cancers [10]. Moreover, in vitro experiments of overexpression or knockdown of FSCN1 revealed that its expression promotes both migration and invasiveness of adenocarcinoma [11-13]or squamous cell carcinoma cell lines [14-16]. Recent research reported that FSCN1 expression in HCCs was associated with loss of E-cadherin, indicating epithelial-mesenchymal transition (EMT)[17]. In their study, the expression levels of FSCN1 and E-cadherin in hepatocellular carcinoma were detected by immunohistochemistry, and they found FSCN1 promoted invasiveness of HCC cells in an MMP-dependent manner. However, the molecular mechanism of FSCN1 in EMT is still not clear. In previous study, we found inhibition of FSCN1 by FSCN1 siRNA blocked EMT in EOC cells [18]. However, few researches are performed to elaborate the mechanism of FSCN1 in EMT. Here we showed that FSCN1 physiologically interacted with and increased the levels of snail1 to promote EMT in ovarian cancer cells.

\section{Materials and Methods}

\section{Cell lines and human tissue specimens}

Human ovarian cancer cell lines SKOV3 (obtained from ATCC, Manassas, VA, USA) and 3AO (purchased from the Chinese Academy of Sciences Type Culture Collection, Shanghai, China) were maintained in RPMI 1640 medium (Gibco-BRL, Gaithersburg, MD, USA) supplemented with $10 \%(\mathrm{v} / \mathrm{v})$ fetal bovine serum, $1 \%$ penicillin antibiotics-antimycotics at $37^{\circ} \mathrm{C}$ under a humidified $5 \% \mathrm{CO} 2$ atmosphere. Human ovarian carcinomas and matched normal ovarian tissue samples were collected from patients at The First Affiliated Hospital of Xi'an Jiaotong University, PR China. This study was approved by the Ethics Committee of The First Affiliated Hospital of Xi'an Jiaotong University, China. Written consent was obtained from each study participant enrolled. The clinicopathologic characteristics of the informative cases are shown in Table 1.

RNA extraction and $q R T-P C R$ analyses

Total RNA was extracted from cells with TRIzol reagent (Invitrogen, Carlsbad, CA, USA). Of note, 1-3 $\mu$ g RNA was reverse transcribed using theRevertAid First Strand cDNA Synthesis Kit (PerfectReal-Time; Takara, Dalian, China), quantitative reverse transcriptase-polymerase chain reaction (qRT-PCR) analyses were performed on the CFX96 Touch

Table 1. Summary of clinical characteristics

\begin{tabular}{lc}
\hline Characteristics & Cases \\
\hline Ovarian cancer tissue & 27 \\
Total number & \\
Median age years(range) & $61(42-75)$ \\
FIGO stage & \\
I/ II & 10 \\
IlyIV & 17 \\
Subtypes of ovarian cancer & \\
Serous cancer & 15 \\
Mucinous & 9 \\
Endometrioid cancer & 2 \\
Clear cell cancer & 1 \\
Normal ovarian tissue & 12 \\
Total number & \\
\hline
\end{tabular}




\section{Cellular Physiology Cell Physiol Biochem 2018;49:1766-1777

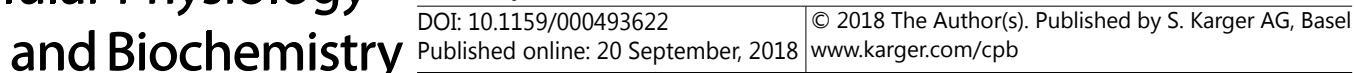

Li et al.: Interaction of FSCN1 and Snail1 in EOC

Real-Time PCR Detection System (Bio-Rad Laboratories, Hercules, CA, USA). The conditions for PCR reaction were as follows: initial denaturation at $95^{\circ} \mathrm{C}$ for $10 \mathrm{~s}$, followed by 40 cycles of $95^{\circ} \mathrm{C}$ for $5 \mathrm{~s}, 57^{\circ} \mathrm{C}$ for $15 \mathrm{~s}$ and $72^{\circ} \mathrm{C}$ for $20 \mathrm{~s} . \Delta \mathrm{Ct}$ values were normalized to $\beta$-actin as the internal control. Each sample was analyzed in triplicate, and all experiments were performed in triplicates. The primer sequences as follows:

E-cadherin forward: 5'-GCTGCTCTTGCTGTTTCTTCG-3';

E-cadherin reverse: 5' -CCGCCTCCTTCTTCATCATAG-3';

FSCN1 forward: 5'-GGCAAGTTTGTGACCTCCAAGAA-3';

FSCN1 reverse: 5'-AGCCGATGAAGCCATGCTC-3';

snail1 forward: 5'-TCCAGAGTTTACCTTCCAGCA-3';

snail1 reverse: 5'-CTTTCCCACTGTCCTCATCTG-3';

vimentin forward: 5'-AAGTTTGCTGACCTCTCTGAGGCT-3';

vimentin reverse: 5'-CTTCCATTTCACGCATCTGGCGTT-3';

$\beta$-actin forward :5' - TCCCTGGAGAAGAGCTACGA-3';

$\beta$-actin reverse :5'- AGCACTGTGTTGGCGTACAG-3'.

\section{Western blot}

Total protein was collected from cells by RIPA lysis buffer containing protease inhibitors (Roche, Indianapolis, IN, USA) and $1 \mathrm{mM}$ PMSF on ice. Protein concentration was measured using the BCA-200 Protein Assay kit (Pierce, Rockford, IL, USA). After heat denaturation at $100{ }^{\circ} \mathrm{C}$ for 5 min, proteins were separated by electrophoresis on $10 \%$ SDS-PAGE gels and then transferred onto nitrocellulose membranes (Pall Life Science, NY, USA). The membranes were blocked with $5 \%$ non-fat milk at room temperature for $1 \mathrm{~h}$, and then incubated overnight at $4{ }^{\circ} \mathrm{C}$ with rabbit anti-human E-cadherin(1:1000, Cell Signaling Technology, Danvers, MA, USA), vimentin(1:500, Cell Signaling Technology, Danvers, MA, USA), FSCN1(1:100000, Abcam, Cambridge, MA, USA), snail(1:300, Cell Signaling Technology, Danvers, MA, USA), and mouse antihuman $\beta$-actin(1:1000, Cell Signaling Technology, Danvers, MA, USA). After washing with TBST, the blots were incubated with horse radish peroxidase (HRP)-conjugated goat anti-rabbit or anti-mouse IgG. Blots were visualized using ECL reagents (Pierce, Rockford, IL, USA) by a chemiluminescence imaging system (Bio-Rad, Richmond, CA, USA).

\section{Plasmid transfection}

The human FSCN1 expression vector pLenti6/V5-DEST-FASCIN was a gift from Lynda Chin (Addgene, \#31207)[19]. SKOV3 and 3AO cells were seeded into 6-well plates until $70 \%-90 \%$ confluency and transiently transfected with pLenti6/V5-DEST-FASCIN or control vector $3 \mu \mathrm{g}$ per well using the X-treme GENE HP DNA Transfection Reagent (Roche, Indianapolis, IN, USA) following the manufacturer's protocol. After 48 hours of transfection, the cells were harvested for further study.

\section{siRNA and transient transfection}

Human FSCN1 siRNA and snail1 siRNA were purchased from GenePharma (Shanghai, China). Ovarian cancer cells were seeded into 6-well plates until they reached $40 \%-50 \%$ confluency. FSCN1 siRNA (GCAGCCTGAAGAAGAAGCA) or snail1 siRNA (AGACCCACUCAGAUGUCAAGAAGUA) was transiently transfected 100nM per well using the X-treme GENE siRNA Transfection Reagent (Roche, Indianapolis, IN, USA). After 48 hours transfection, the cells were harvested for further studies.

\section{Cell migration and invasion assay}

After treated, cells were trypsinized and counted. A total of $1 \times 10^{5}$ cells (for migration assay) or $5 \times 10^{5}$ cells (for invasion assay) in $100 \mu \mathrm{l}$ serum-free medium were added into millicells (Millipore Co., Bedford, MA, USA) without (for migration assay) or with (for invasion assay) Matrigel (Becton Dickinson Labware, Bedford, MA, USA) coated. $500 \mu \mathrm{l}$ of 1640 medium containing $20 \%$ newborn bovine serum was added to the bottom chambers as the chemotactic factor. After incubation for $24 \mathrm{~h}$ (for migration assay) or $48 \mathrm{~h}$ (for invasion assay) at $37^{\circ} \mathrm{C}$, cells remaining on the upper surface of the filter were removed using cotton swabs. Then the migrated cells were fixed using methyl alcohol and stained using $0.1 \%$ crystal violet. Migratory (or invasive) cells were counted and averaged from images of five random fields (original magnification $\times 200$ ) captured using an inverted light microscope. The mean values of three duplicate assays were used for statistical analysis. 


\section{Cellular Physiology Cell Physiol Biochem 2018;49:1766-1777

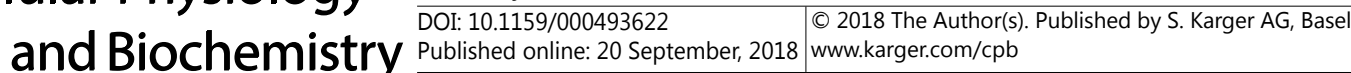 \\ Li et al.: Interaction of FSCN1 and Snail1 in EOC}

\section{Co-Immunoprecipitation}

Co-IP assays were carried out using Pierce crosslink immunoprecipitation kits (Thermo Scientific, Rockford, IL, USA) as per the manufacturer's instructions. Cells were treated with $1 \mathrm{ml}$ of extraction buffer (10 mmol/l HEPEs, pH7.5, $100 \mathrm{mmol} / \mathrm{l} \mathrm{NaCl}, 1 \mathrm{mmol} / \mathrm{l}$ EDTA, 10\% Glycerol, 0.5\% Triton X-100 and $5 \mu \mathrm{mol} / \mathrm{l}$ MG132). Co-IP procedures were performed at $4{ }^{\circ} \mathrm{C}$ unless otherwise indicated, using a Pierce spin column which can be capped and plugged with a bottom plug for incubation or unplugged to remove the supernatant by centrifugation at $1000 \mathrm{~g}$ for 1 minute. The binding of the first antibody to protein A/G agarose was performed with the protocol described in Pierce crosslink immunoprecipitation kits (Thermo Scientific, Rockford, IL, USA) with slight modification. For co-IP experiment without using DSS crosslinking, the protein $\mathrm{A} / \mathrm{G}$ agarose was incubated anti-FSCN 1 antibody at $25^{\circ} \mathrm{C}$ for $1 \mathrm{~h}$ on a mixer, followed by incubation with $600 \mu \mathrm{l}$ pre-cleared lysate overnight. The immunoprecipitated products were washed with the washing buffer five times and eluted with $2 \mathrm{X}$ Laemmli buffer at $100^{\circ} \mathrm{C}$ for $10 \mathrm{~min}$. The cap of the spin column was loose to avoid overpressure and leakage from the bottom when boiling. The eluting complex was subjected to SDS-PAGE separation for Western blot.

\section{Statistical analysis}

All experiments were performed at least in triplicate, and each experiment was independently performed at least 3 times. The graphical presentations were performed using GraphPad Prism 5.0. Data were presented as the means \pm SE and were analyzed using SPSS 22.0 software (Chicago, IL, USA). Statistical differences were tested by Chi-square test or two-tailed t-test. Differences were considered significant at $\mathrm{P}$ $<0.05\left(^{*}\right)$ or highly significant at $\mathrm{P}<0.001\left(^{* *}\right)$.

\section{Results}

FSCN1 and snail1 relative level was positively correlated in ovarian cancer tissues and cell lines

In order to compare the expression of FSCN1 and snail1 in EOC, we detected mRNA levels of FSCN1 and snail 1 in 12 normal ovarian tissues and 27 epithelial ovarian cancer tissues. Total RNA was extracted from these samples, quantitative real-time PCR analysis was performed to detect the mRNA expression level of FSCN1 and snail1. We identified that FSCN1 and snail 1 level in ovarian cancer tissues were higher than in normal ovarian tissues (Fig. 1A, C). Moreover, the results showed the more late the clinical period, the higher the expression of FSCN1 (Fig. 1B). Moreover, by comparing the relationship of RNA expression levels between FSCN1 and snail1 in ovarian cancer tissues, we found that the mRNA expression of snail1 was positively correlated with FSCN1 in ovarian cancer tissues (Fig. 1D). We identified the differential expression of FSCN1 among normal ovarian and EOC cell lines, SKOV3 and 3AO. the results showed that the snail1 level in FSCN1 high-expression cell line (SKOV3) was higher than FSCN1 low-expression cell line (3AO) (Fig. 1E). These results demonstrated that mRNA expression levels of FSCN1 are increased in ovarian cancer tissues and positively correlated with clinical stages.

\section{Overexpression of FSCN1 promoted EMT in ovarian cancer cells}

We manipulated FSCN1 expression in ovarian cancer cells to analyze its association with EMT. With forced expression of FSCN1, EMT phenomenon was observed in SKOV3 and $3 \mathrm{AO}$ cells, as showen by decreased expression of E-cadherin, increased expression of vimentin(Fig. 2A, B), Overexpression of FSCN1 promoted migration(Fig. 2C) and invasion(Fig. 2D) of ovarian cancer cells. Taken together, our data suggested that FSCN1 was required for EMT of ovarian cancer cells. 


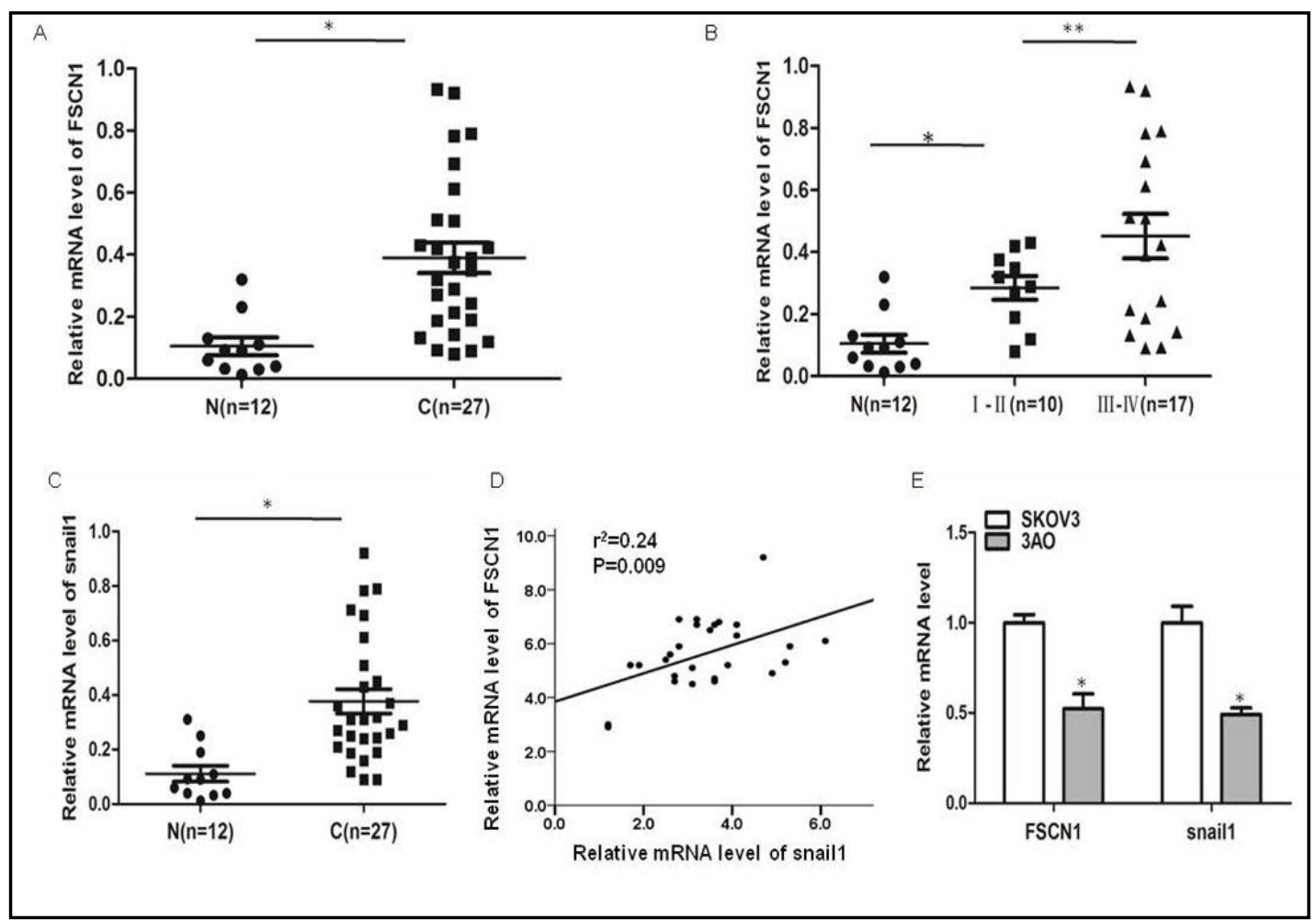

Fig. 1. FSCN1 was increased in ovarian cancer tissues and positively correlated with clinical stages.(A) mRNA level of FSCN1 in ovarian cancer tissues(n=27) was higher than in the normal ovarian tissues $(n=12)$. (B) Relative expression of FSCN1 in ovarian cancer tissues (III/IV) ( $n=17)$ was higher than that in ovarian cancer tissues (I/II) $(n=10)$. (C) mRNA level of snail1 in ovarian cancer tissues( $n=27)$ was higher than in the normal ovarian tissues(n=12). (D) Scatter diagram showing snail1 expression and FSCN1 expressions by qPCR and their correlations( $\mathrm{r} 2=0.24, \mathrm{P}=0.009)$ in 27 tissues of EOC. (E) Quantitative real-time PCR results showed that the expression of FSCN1 and snail was higher in SKOV3 cells than in 3AO cells; All experiments were carried out in triplicate and the results were presented as means $\pm \mathrm{SE}$. ${ }^{*} \mathrm{P}<0.05,{ }^{* *} \mathrm{P}<0.001$, $\mathrm{t}$-test.

\section{Downexpression of FSCN1 blocked EMT in ovarian cancer cells}

In previous study [18], we used FSCN1 siRNA decreased FSCN1 expression in ovarian cancer cells. EMT was reversed in SKOV3 and 3AO cells by downregulation of FSCN1, as shown by increased expression of E-cadherin, decreased expression of vimentin (Fig. 3A, B), and demotion of migration (Fig. 3C) and invasion (Fig. 3D). Taken together, our data suggested that downexpression of FSCN1 blocked EMT in ovarian cancer cells.

\section{FSCN1 promoted snail1 in ovarian cancer cells}

Transcription factors that repress E-cadherin are considered as EMT-promoting factors, including snail1, slug, ZEB1, and so on. Snail1 has a pivotal role in the regulation of EMT, involving the loss of E-cadherin and claudins with concomitant upregulation of vimentin and fibronectin, among other biomarkers $[7,8]$. To examine whether FSCN1 may regulate the levels of snail1 in EOC cells, we then transfected the EOC cells with either a FSCN1overexpressing plasmid, or a small interfering RNA(siRNA) for FSCN1(siFSCN1), to increase or decrease FSCN1 levels in EOC cells, respectively. The changes of FSCN1 in EOC cells were confirmed by RT-qPCR (Fig. 4A, C), and by Western blot (Fig. 4B, D). We found that neither FSCN1 overexpression nor depletion significantly affected the transcript levels of snail1(Fig. 4A, C). However, FSCN1 overexpression significantly increased the protein level of snail1 in EOC cells (Fig. 4B), while depletion of FSCN1 significantly decreased the protein level of snail1 in EOC cells (Fig. 4D). 


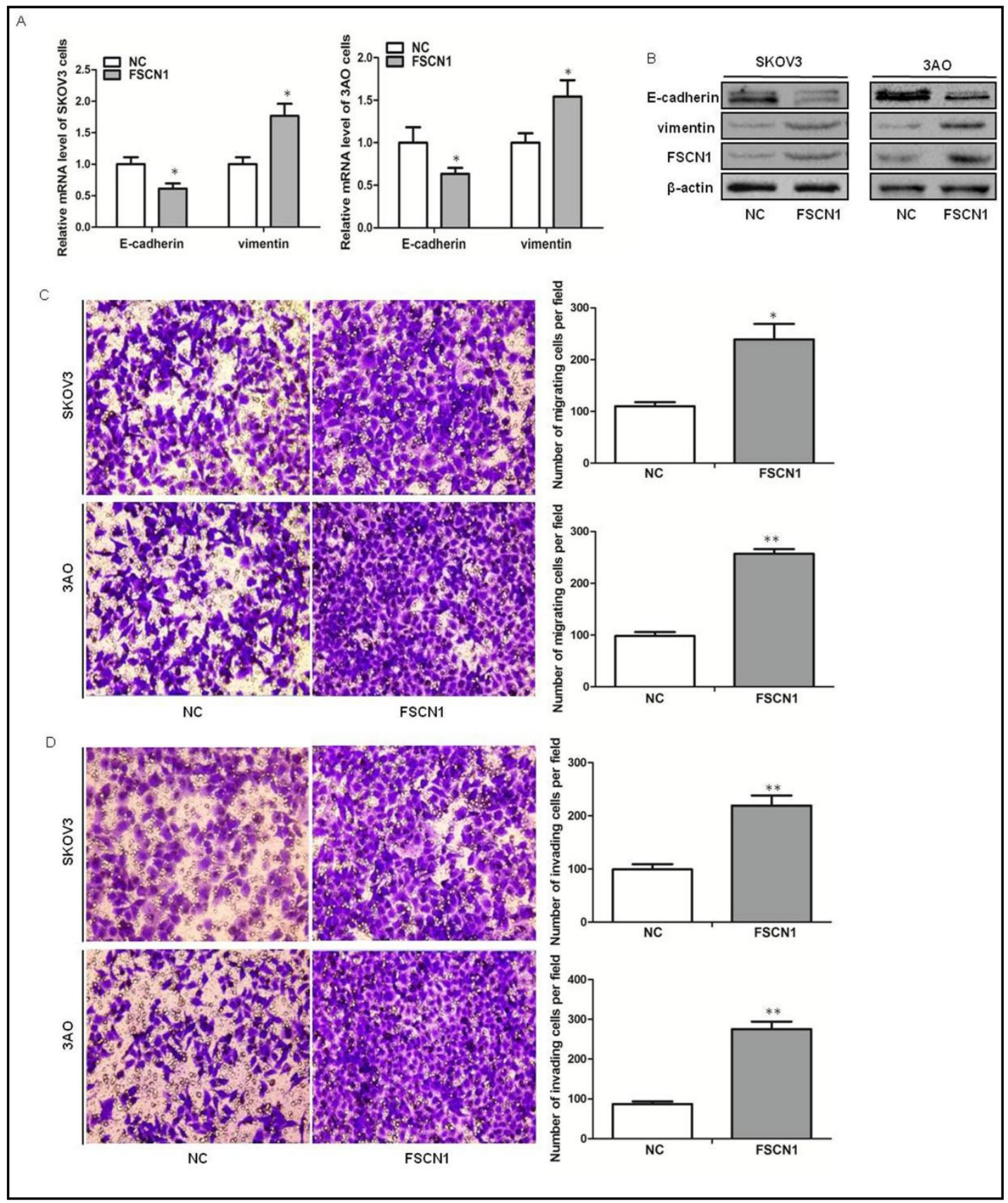

Fig. 2. Overexpression of FSCN1 promoted EMT in ovarian cancer cells. (A) Quantitative real-time PCR showed that E-cadherin was deceased whereas vimentin was increased at mRNA level in cells by overexpression of FSCN1.(B) Western blot assays showed that E-cadherin was decreased whereas vimentin was increased at protein level in cells by overexpression of FSCN1.(C) In a transwell migration assay, plasmid vector-mediated FSCN1 overexpression in SKOV3 and 3AO cells increased the cell invading capacity( The migrated cells were fixed, stained and counted $24 \mathrm{~h}$ later). (D)In a transwell invasion assay, plasmid vectormediated FSCN1 overexpression in SKOV3 and 3AO cells increased the cell invading capacity (The invased cells were fixed, stained and counted $48 \mathrm{~h}$ later) All of the treatments in this figure were carried out in triplicate, and the results are displayed as the means $\pm \mathrm{SD} .{ }^{*} \mathrm{P}<0.05,{ }^{* *} \mathrm{P}<0.01$, t-test. 


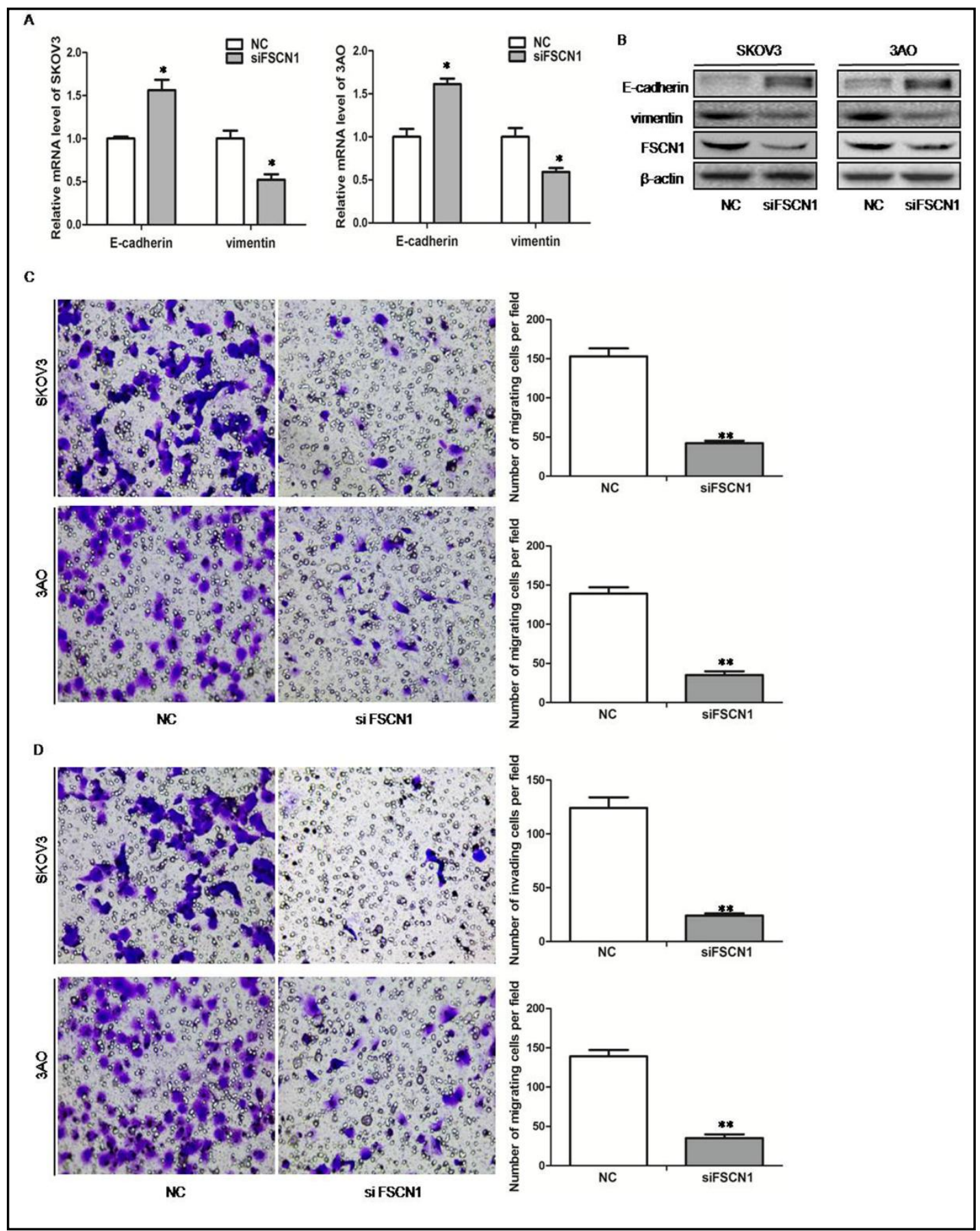

Fig. 3. Downexpression of FSCN1 blocked EMT in ovarian cancer cells. (A) Quantitative real-time PCR showed that E-cadherin was increased whereas vimentin was decreased at mRNA level in cells by downregulation of FSCN1.(B) Western blot assays showed that E-cadherin was inceased whereas vimentin was decreased at protein level in cells by downexpression of FSCN1.(C) In a transwell migration assay, FSCN1 siRNA-mediated FSCN1 downexpression in SKOV3 and 3AO cells decreased the cell invading capacity( The migrated cells were fixed, stained and counted $24 \mathrm{~h}$ later). (D) In a transwell invasion assay, FSCN1 siRNA-mediated FSCN1 downexpression in SKOV3 and 3AO cells decreased the cell invading capacity (The invased cells were fixed, stained and counted $48 \mathrm{~h}$ later) All of the treatments in this figure were carried out in triplicate, and the results are displayed as the means $\pm \mathrm{SD}$. ${ }^{*} \mathrm{P}<0.05,{ }^{* *} \mathrm{P}<0.01$, t-test. 


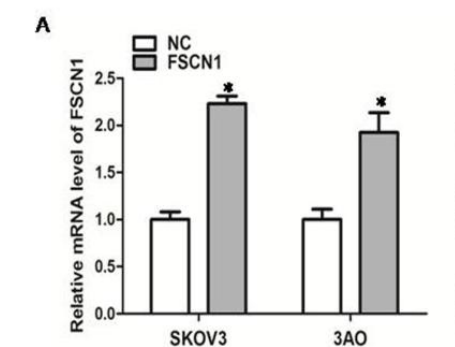

c

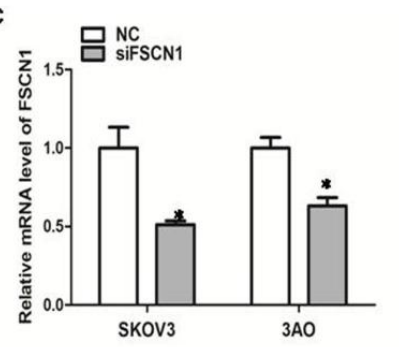

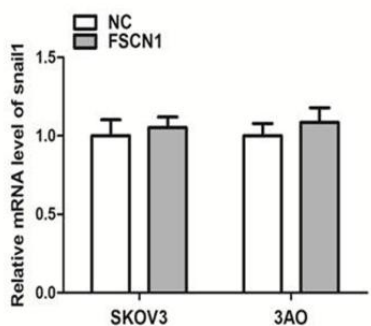

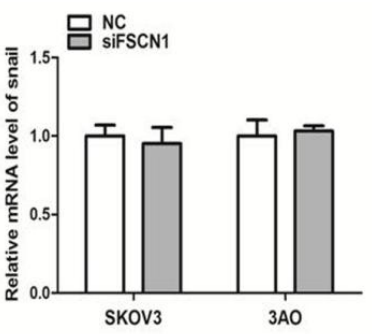

B

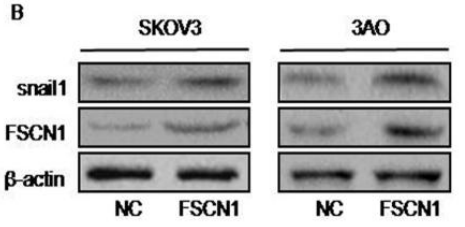

D
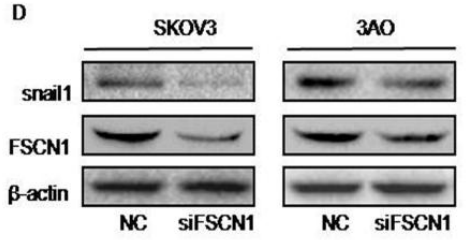

Fig. 4. FSCN1 promoted snail1 in ovarian cancer cells. (A)Quantitative real-time PCR showed that overexpression of FSCN1 didn't affect the transcript levels of snail1.(B)Western blot assays showed that overexpression of FSCN1 promoted snail1 in protein level.(C)Quantitative real-time PCR showed that downexpression of FSCN1 didn't affect the transcript levels of snail1. (D)Western blot assays showed that downexpression of FSCN1 decreased snail1 in protein level. All of the treatments in this figure were carried out in triplicate, and the results are displayed as the means $\pm \mathrm{SD} .{ }^{*} \mathrm{P}<0.05,{ }^{* *} \mathrm{P}<0.01$, t-test.

FSCN1 physiologically associated with snail1 in ovarian cancer cells

To confirm the specific binding between FSCN1 and snail1, we performed immunoprecipitation (IP) to detect the interaction between endogenous proteins in two EOC lines, SKOV3 and 3AO. Our results showed that endogenous snail1 co-precipitated with FSCN1 in both lines (Fig. 5). These data suggest that FSCN1 and snail1 physiologically associate in EOC cells.

FSCN1 promotes EMT through snail1 in ovarian cancer cells

We further investigated whether FSCN1 mediated the effect of snail 1 on EMT. In cells co-transfected with pLenti6/V5-DEST-FASCIN and snail1 siRNA, the effect of FSCN1 induced EMT as evidenced by downregulation of E-cadherin, upregulation of vimentin (Fig. 6A, B), and accumulation of migration (Fig. 6C) and invasion (Fig. 6D) was largely reversed by ectopic downexpression of snail1. Notably, snail1 had no significant influence on FSCN1 expression(Fig. 6A, B). Taken together, our data suggested that FSCN1 induced EMT by targeting snail1 in ovarian cancer cells.

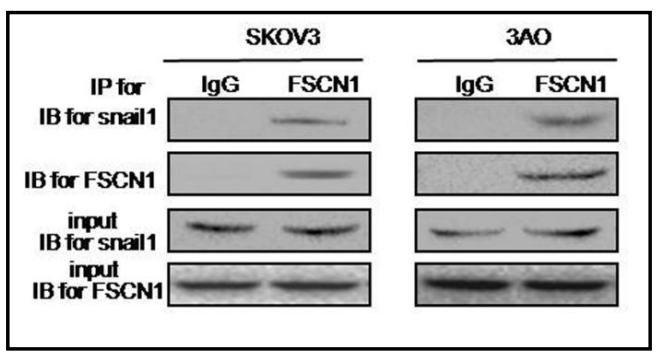

Fig. 5. FSCN1 physiologically associated with snail1 in ovarian cancer cells. CoImmunoprecipitation (Co- IP) was performed to detect the interaction between endogenous proteins independently in two EOC lines, SKOV3 and $3 A 0$. IgG antibody was used as negative control of immunoprecipitation (IP) and $25 \mu \mathrm{g}$ whole cell lysate was used as input. Our results showed that endogenous snail1 co-precipitated with FSCN1 in both lines. IB: immunoblot. IP: immunoprecipitation. All of the treatments in this figure were carried out in triplicate, and the results are displayed as the means $\pm \mathrm{SD}$. ${ }^{*} \mathrm{P}<0.05$, ${ }^{* *} \mathrm{P}<0.01$, t-test. 


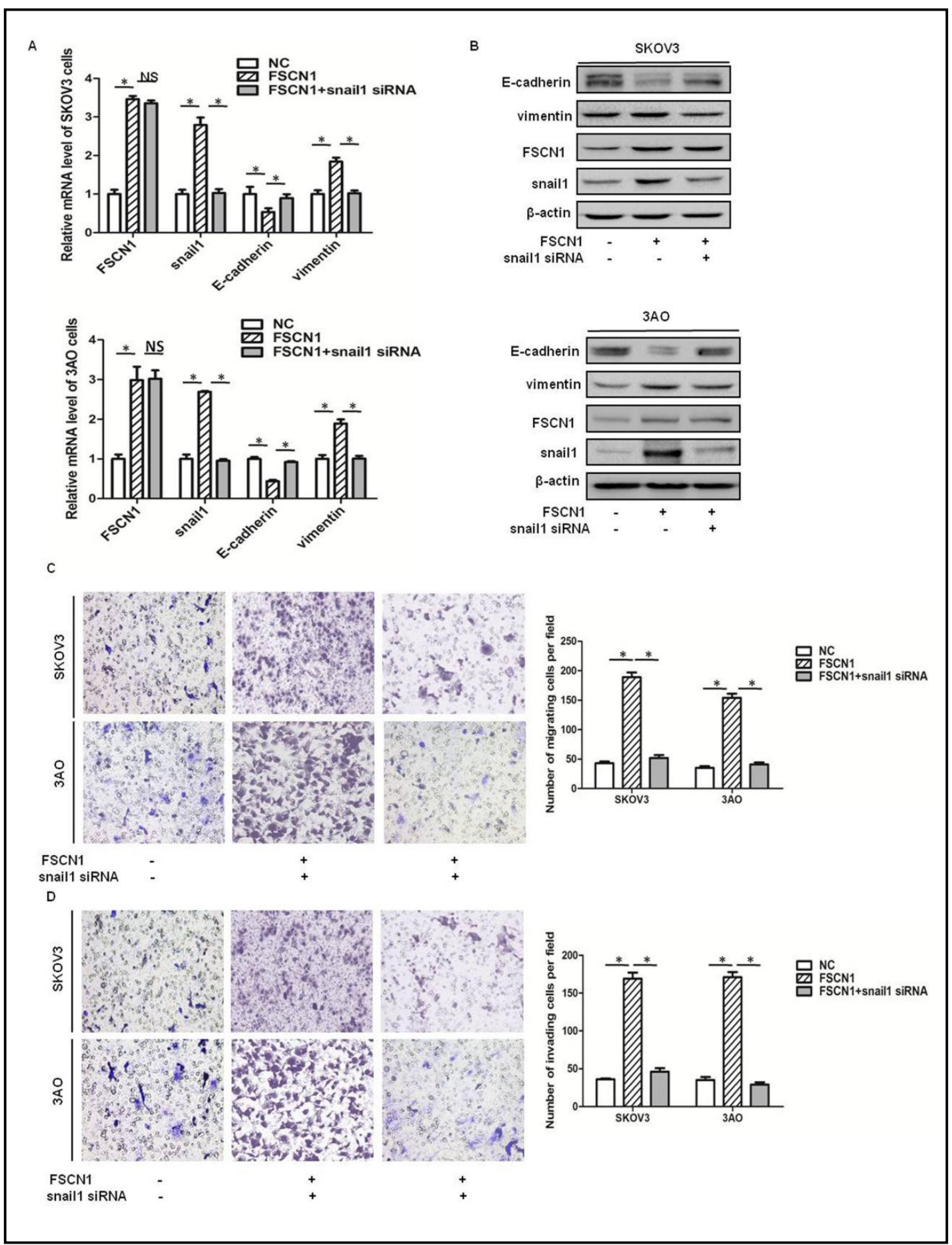

Fig. 6. FSCN1 promotes EMT through snail1 in ovarian cancer cells. (A)Quantitative real-time PCR showed that downexpression of snail1 attenuated the E-cadherin downregulation and vimentin upregulation caused by FSCN1 transfection. (B)Western blot assays showed that downexpression of snail1 increased the E-cadherin downregulation and vimentin upregulation caused by FSCN1 transfection. (C)In a transwell migration assay, downexpression of snail1 largely counteracted FSCN1 accumulation of the migration. (D) In a transwell invasion assay, downexpression of snail1 largely counteracted FSCN1 accumulation of the invasion. (The invased cells were fixed, stained and counted $48 \mathrm{~h}$ later). Images were photographed using a phase contrast microscopy $(200 \times)$. All of the treatments in this figure were carried out in triplicate, and the results are displayed as the means $\pm \mathrm{SD} .{ }^{*} \mathrm{P}<0.05,{ }^{* *} \mathrm{P}<0.01$, t-test. 


\section{Cellular Physiology Cell Physiol Biochem 2018;49:1766-1777

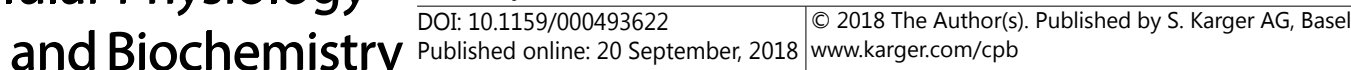 \\ Li et al.: Interaction of FSCN1 and Snail1 in EOC}

\section{Discussion}

The high malignancy and lethality of EOC largely result from its high invasiveness and local and distal metastasis, in which EMT plays a substantial role. Emerging evidence shows that epithelial-mesenchymal transition (EMT) plays a crucial role in metastatic dissemination of EOC [20]. Characterized by the molecular events including loss of epithelial cell-specific markers such as E-cadherin and ZO-1, gain of mesenchymal cell-specific markers like vimentin and $\mathrm{N}$-cadherin, and increase of a series of transcription factors that repress E-cadherin transcription [21-23]. Among all EMT-associated proteins, snail1 is a potential EMT activator, and directly inhibited E-cadherin.

FSCN1 was an actin-bundling protein functioning in cell movement under physiological or pathological conditions $[10,24]$. Overexpression of FSCN1 promoted migration and invasion of cancer cells [11, 12,14-16, 25-27], and was associated with clinically unfavorable phenotypes in human epithelial cancers including EOC $[10,17,27,28]$. Nevertheless, the correlation of FSCN1 with EMT was unkown. Here we provided the evidence that overexpression of FSCN1 was sufficient to confer EMT in ovarian cancer cells, and its aberrant elevation in ovarian cancer increased snail 1 expression. Snail 1 is recognized as a transcription factor in EMT, therefore, we did not study the regulatory role of snail1 on EMT.

In our study, our data compellingly showed that FSCN1 significantly increased in EOC, and especially in EOC with metastasis. These data provide a clinical relevance of the current study. Moreover, we further showed that FSCN1 physiologically interacted with and increased the levels of snail 1 to promote ovarian cancer cell invasiveness, and the promotion of FSCN1 to EMT was mediated by snail1. Notably, FSCN1 could promoted snail expression, but snail1 had no significant influence on FSCN1 expression, so snail is the downstream factor of FSCN1.

Together with previous reports by us and by others, a comprehensive regulatory network on EMT, highlighting a pivotal role of FSCN1, has been proposed. The precise mechanism of FSCN1 in EMT process has not been clearly elucidated. In some cases, high FSCN1 expression has been correlated with low E-cadherin expression, they gain FSCN1 whilst losing E-cadherin. There was evidence that fascin 1 expression was regulated by two pathways, the WNT activated TCF/LEF (T cell factor/lymphocyte enhancer-binding factor) transcriptional signaling pathway that promoted EMT and cyclic-AMP response element binding protein (CREB) and the arylhydrocarbon receptor (AhR) $[29,30]$. Studys have reported that FSCN1 primarily acts as a migration factor associated with EMT in HCC cells and facilitates their invasiveness in combination with MMPs, but the relationship between these factors and EMT has yet to be determined [17]. Fouthermore, the report demonstrated FSCN1 is a downstream effector of SNAI2 in promoting EMT in HNSC cells [31]. In our study, we found that FSCN1 physiologically interacted with and increased the levels of snail1 to promote EMT in ovarian cancer cells. The result is very meaningful for establishing the functional network of FSCN1 in EMT.

\section{Conclusion}

In conclusion, we have demonstrated that FSCN1 expression was significantly correlated with advanced clinical stages, and we showed that FSCN1 physiologically interacted with and increased the level of snail 1 to promote EMT in ovarian cancer cells. Our results indicate a mechanism of EMT in ovarian cancer. Thus, our study sheds light on FSCN1 as a promising therapeutic target for EOC. 


\section{Cellular Physiology Cell Physiol Biochem 2018;49:1766-1777 \begin{tabular}{l|l|l|l|l} 
O 2018 The Author(s). Published by S. Karger AG, Basel \\
and Biochemistry
\end{tabular}

\section{Acknowledgements}

This work was supported by National Natural Science Foundation of China (No. 81702577 and No. 81202373).

\section{Disclosure Statement}

The authors declare that no potential conflicts of interest exist.

\section{References}

1 Siegel R, Ma J, Zou Z, Jemal A: Cancer statistics, 2014. CA Cancer J Clin 2014;64:9-29.

-2 Elzek MA, Rodland KD: Proteomics of ovarian cancer: functional insights and clinical applications. Cancer Metastasis Rev 2015;34:83-96.

-3 Shang Y, Cai X, Fan D: Roles of epithelial-mesenchymal transition in cancer drug resistance. Curr Cancer Drug Targets 2013;13:915-929.

4 Kiesslich T, Pichler M, Neureiter D: Epigenetic control of epithelial-mesenchymal-transition in human cancer. Mol Clin Oncol 2013;1:3-11.

5 Meng F, Wu G: The rejuvenated scenario of epithelial-mesenchymal transition (EMT) and cancer metastasis. Cancer Metastasis Rev 2012;31:455-467.

6 Tan H, He Q Gong G, Wang Y, Li J, Wang J, Zhu D, Wu X: miR-382 inhibits migration and invasion by targeting ROR1 through regulating EMT in ovarian cancer. Int J Oncol 2016;48:181-190.

7 Kaufhold S, Bonavida B: Central role of Snail1 in the regulation of EMT and resistance in cancer: a target for therapeutic intervention. J Exp Clin Cancer Res 2014;33:62.

-8 Franco DL, Mainez J, Vega S, Sancho P, Murillo MM, de Frutos CA, Del Castillo G, Lopez-Blau C, Fabregat I, Nieto MA: Snail1 suppresses TGF-beta-induced apoptosis and is sufficient to trigger EMT in hepatocytes. J Cell Sci 2010;123:3467-3477.

-9 Chen L, Yao Y, Sun L, Zhou J, Miao M, Luo S, Deng G, Li J, Wang J, Tang J: Snail Driving Alternative Splicing of CD44 by ESRP1 Enhances Invasion and Migration in Epithelial Ovarian Cancer. Cell Physiol Biochem 2017;43:2489-2504.

10 Hashimoto Y, Skacel M, Adams JC: Roles of fascin in human carcinoma motility and signaling: prospects for a novel biomarker? Int J Biochem Cell Biol 2005;37:1787-1804.

11 Jawhari AU, Buda A, Jenkins M, Shehzad K, Sarraf C, Noda M, Farthing MJ, Pignatelli M, Adams JC: Fascin, an actin-bundling protein, modulates colonic epithelial cell invasiveness and differentiation in vitro. Am J Pathol 2003;162:69-80.

12 Hashimoto Y, Parsons M, Adams JC: Dual actin-bundling and protein kinase C-binding activities of fascin regulate carcinoma cell migration downstream of Rac and contribute to metastasis. Mol Biol Cell 2007;18:4591-4602.

13 Darnel AD, Behmoaram E, Vollmer RT, Corcos J, Bijian K, Sircar K, Su J, Jiao J, Alaoui-Jamali MA, Bismar TA: Fascin regulates prostate cancer cell invasion and is associated with metastasis and biochemical failure in prostate cancer. Clin Cancer Res 2009;15:1376-1383.

14 Hashimoto Y, Ito T, Inoue H, Okumura T, Tanaka E, Tsunoda S, Higashiyama M, Watanabe G, Imamura M, Shimada Y: Prognostic significance of fascin overexpression in human esophageal squamous cell carcinoma. Clin Cancer Res 2005;11:2597-2605.

-15 Xie JJ, Xu LY, Zhang HH, Cai WJ, Mai RQ Xie YM, Yang ZM, Niu YD, Shen ZY, Li EM: Role of fascin in the proliferation and invasiveness of esophageal carcinoma cells. Biochem Biophys Res Commun 2005;337:355-362.

16 Chen SF, Lin CY, Chang YC, Li JW, Fu E, Chang FN, Lin YL, Nieh S: Effects of small interfering RNAs targeting Fascin on gene expression in oral cancer cells. J Oral Pathol Med 2009;38:722-730. 


\section{Cellular Physiology Cell Physiol Biochem 2018;49:1766-1777

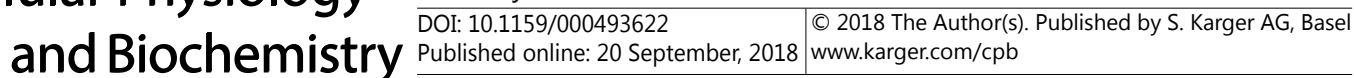 \\ Li et al.: Interaction of FSCN1 and Snail1 in EOC}

17 Hayashi Y, Osanai M, Lee GH: Fascin-1 expression correlates with repression of E-cadherin expression in hepatocellular carcinoma cells and augments their invasiveness in combination with matrix metalloproteinases. Cancer Sci 2011;102:1228-1235.

18 Li J, Lu J, Ye Z, Han X, Zheng X, Hou H, Chen W, Li X, Zhao L: 20(S)-Rg3 blocked epithelial-mesenchymal transition through DNMT3A/miR-145/FSCN1 in ovarian cancer. Oncotarget 2017;8:53375-53386.

19 Scott KL, Nogueira C, Heffernan TP, van Doorn R, Dhakal S, Hanna JA, Min C, Jaskelioff M, Xiao Y, Wu CJ, Cameron LA, Perry SR, Zeid R, Feinberg T, Kim M, Vande Woude G, Granter SR, Bosenberg M, Chu GC, DePinho RA et al.: Proinvasion metastasis drivers in early-stage melanoma are oncogenes. Cancer Cell 2011;20:92-103.

20 Ying X, Wei K, Lin Z, Cui Y, Ding J, Chen Y, Xu B: MicroRNA-125b Suppresses Ovarian Cancer Progression via Suppression of the Epithelial-Mesenchymal Transition Pathway by Targeting the SET Protein. Cell Physiol Biochem 2016;39:501-510.

-21 Cho KR, Shih Ie M: Ovarian cancer. Annu Rev Pathol 2009;4:287-313.

-22 Swisher EM, Taniguchi T, Karlan BY: Molecular scores to predict ovarian cancer outcomes: a worthy goal, but not ready for prime time. J Natl Cancer Inst 2012;104:642-645.

23 Huang RY, Chung VY, Thiery JP: Targeting pathways contributing to epithelial-mesenchymal transition (EMT) in epithelial ovarian cancer. Curr Drug Targets 2012;13:1649-1653.

24 Liu Z, Ning G, Xu R, Cao Y, Meng A, Wang Q: Fscn1 is required for the trafficking of TGF-beta family type I receptors during endoderm formation. Nat Commun 2016;7:12603.

25 Zhang M, Zhao Z, Duan X, Chen P, Peng Z, Qiu H: FSCN1 predicts survival and is regulated by a PI3Kdependent mechanism in renal cell carcinoma. J Cell Physiol 2017;10.1002/jcp.26264.

26 Zhang N, Bi X, Zeng Y, Zhu Y, Zhang Z, Liu Y, Wang J, Li X, Bi J, Kong C: TGF-beta1 promotes the migration and invasion of bladder carcinoma cells by increasing fascin1 expression. Oncol Rep 2016;36:977-983.

-27 Wang CQ Tang CH, Wang Y, Jin L, Wang Q Li X, Hu GN, Huang BF, Zhao YM, Su CM: FSCN1 gene polymorphisms: biomarkers for the development and progression of breast cancer. Sci Rep 2017;7:15887.

28 Park SH, Song JY, Kim YK, Heo JH, Kang H, Kim G, An HJ, Kim TH: Fascin1 expression in high-grade serous ovarian carcinoma is a prognostic marker and knockdown of fascin 1 suppresses the proliferation of ovarian cancer cells. Int J Oncol 2014;44:637-646.

-29 Vignjevic D, Schoumacher M, Gavert N, Janssen KP, Jih G, Lae M, Louvard D, Ben-Ze'ev A, Robine S: Fascin, a novel target of beta-catenin-TCF signaling, is expressed at the invasive front of human colon cancer. Cancer Res 2007;67:6844-6853.

-30 Hashimoto Y, Loftis DW, Adams JC: Fascin-1 promoter activity is regulated by CREB and the aryl hydrocarbon receptor in human carcinoma cells. PLoS One 2009;4:e5130.

-31 Wang L, Jia Y, Jiang Z, Gao W, Wang B: FSCN1 is upregulated by SNAI2 and promotes epithelial to mesenchymal transition in head and neck squamous cell carcinoma. Cell Biol Int 2017;41:833-841. 